\title{
Estudio serológico frente a los virus respiratorios en terneros en un sistema semi-intensivo y su relación con la sintomatología respiratoria
}

\section{Serological study against respiratory viruses in calves in a semi-intensive system and its relationship to respiratory symptoms}
N. C. Gaeta ${ }^{1}$ 0000-0001-6397-605X
B. L. M. Ribeiro ${ }^{1}$ 0000-0002-4303-0504
B. A. F. D. Oliveira1 0000-0001-7183-3876
M. A. R. Alemán ${ }^{1}$ 0000-0002-5687-7763
C. P. Ribeiro ${ }^{2}$ 0000-0001-8772-5714
L. Gregory ${ }^{1}$ 0000-0003-0240-3025
${ }^{1}$ Departamento de Clínica Médica, Faculdade de Medicina Veterinária e Zootecnia, Universidade de São Paulo. Av. Prof. Orlando Marques de Paiva, 87, Cidade Universitária, São Paulo, Brazil, CEP 05508-270. Email: natalia.gaeta@usp.br
${ }^{2}$ Laboratório de Produção de Imunobiológicos. Instituto Biológico. Av. Conselheiro Rodrigues Alves, 1252, Vila Mariana, São Paulo, Brazil, CEP: 04014-002.

\section{Veterinaria (Montevideo) Volumen 55} No 212 (2019) 66-70

\section{Resumen}

Las enfermedades respiratorias de los terneros son un problema sanitario importante debido a que cursan con altas tasas de morbilidad y mortalidad, originando grandes pérdidas económicas. El objetivo de este estudio fue determinar la presencia de anticuerpos séricos frente a los principales agentes etiológicos virales relacionados con la bronconeumonía en bovinos (virus de la diarrea viral bovina, herpesvirus bovino tipo1 y virus respiratorio sincitial bovino), evaluando la asociación entre la presencia de anticuerpos y las manifestaciones clínicas. Se estudiaron 63 terneros pertenecientes a dos rebaños del estado de Sao Paulo (Brasil). Los animales fueron divididos en dos grupos: animales sanos y enfermos con sintomatología respiratoria después del examen físico. Se extrajo sangre para la posterior detección de anticuerpos en suero por neutralización viral y se estudió la asociación entre los signos clínicos y los hallazgos serológicos. Los anticuerpos frente al virus de la diarrea viral bovina, herpesvirus bovino tipo-1 y virus respiratorio sincitial bovino se detectaron respectivamente en el 57,10\%, el 66,70\% y el $93,70 \%$ de las muestras. Se observó una mayor frecuencia de anticuerpos contra el virus de la diarrea viral bovina en animales con exudado nasal seroso / mucoso $(\mathrm{P}=0,01)$. Los animales deshidratados $(\mathrm{P}=0,008)$ y aquellos con temperatura rectal por encima de $39,5^{\circ} \mathrm{C}(\mathrm{P}=0,03)$ presentaron con mayor frecuencia anticuerpos frente al virus respiratorio sincitial bovino. El presente trabajo presentó resultados de seropositividad más elevados a los encontrados en otros estudios realizados en granjas con un sistema de manejo extensivo, confirmando la importancia del manejo en la transmisión de estos microorganismos. Palabras clave: Virus de la diarrea viral bovina, Herpesvirus bovino tipo-1, Virus respiratorio sincitial bovino, Serología, Neumonía
Recibido: 28/05/2019

Aceptado: 08/07/2019

\section{Summary}

Respiratory diseases are a major health problem due to the high morbidity and mortality rates, with great economic losses. The aim of this study was to determine the frequency of antibodies against the main viral etiological agents related to bronchopneumonia in cattle (bovine viral diarrhea virus, bovine herpesvirus type-1 and bovine respiratory syncytial virus), searching for associations between these agents and the clinical signs observed. We studied 63 calves from two herds from São Paulo, Brazil. After physical examination, the calves were divided into healthy calves and calves with respiratory disease. Serum was obtained for subsequent detection of antibodies by viral neutralization. The association between clinical signs and serological findings was studied. Antibodies against bovine viral diarrhea virus, bovine herpesvirus type-1 and bovine respiratory syncytial virus were detected in $57.10 \%, 66.70 \%$ and $93.70 \%$ of the samples. A higher frequency of antibodies against bovine viral diarrhea virus was observed in animals with serous / mucous nasal discharge $(\mathrm{P}=0.01)$. The dehydrated animals $(\mathrm{P}=0.008)$ and those with a rectal temperature above $39.5^{\circ} \mathrm{C}(\mathrm{P}=0.03)$ presented a higher frequency of antibodies against the bovine respiratory syncytial virus. The present work presented results superior to those found in studies in farms with extensive system, confirming the importance of management in the transmission of microorganisms.

Keywords: Bovine viral diarrhea virus, Bovine herpesvirus type-1, Bovine respiratory syncytial virus, Serology, Pneumonia. 


\section{Introducción}

Las enfermedades respiratorias de los bóvidos o síndrome respiratorio bovino (SRB) son una de las principales causas de pérdidas económicas en los rebaños lecheros, debido a los altos gastos de tratamiento, descarte de la leche, pérdida de peso, retraso en el crecimiento y muerte de los animales enfermos (Miles, 2009; USDA, 2013).

Los agentes virales son considerados agentes primarios y factores determinantes para las infecciones bacterianas secundarias, ya que alteran el funcionamiento de las células del sistema inmunitario, de la mucosa respiratoria y la producción de citocinas (Bosch et al., 2013), entre los patógenos relacionados con las SRB. Los virus de la diarrea vírica bovina (VDVB), el herpesvirus bovino tipo 1 (HVBo-1), el virus respiratorio sincitial bovino (VRSB)y el vírus de parainfluenza tipo 3 (PI-3) se incluyen entre la etiología de este problema, siendo considerados como los principales agentes víricos (Brito et al., 2010; Affonso et al., 2011; Gaeta et al.,2017).

El VDVB pertenece a la familia Flaviviridae y está relacionado con otras patologías como diarrea, enfermedad de las mucosas y abortos (Lanyon et al., 2014; OIE, 2015). El VDVB causa inmunosupresión en los animales, permitiendo el fácil establecimiento de infecciones bacterianas secundarias (Lanyon et al., 2014). La presencia de animales persistentes infectados por VDVB es una de las consecuencias más importantes de la infección de los rebaños por esto virus. La infección de la vaca en el primer trimestre de la gestación por una variante no citopatica del virus resulta en la inhibición de la producción de interferón tipo 1, permitiendo la supervivencia del virus en el feto. Así, al nacer, los animales se convierten en fuentes de infección, con eliminación de gran cantidad de virus en todas las secreciones del animal (Lanyon et al., 2014).

El HVBo-1 pertenece a la familia Herpesviridae y tiene como principal característica una fase de latencia en la que el animal puede eliminar el virus después de situaciones estresantes como transporte, destete e confinamiento, lo que proporciona una fuente de infección silenciosa en el rebaño. Los animales pueden presentar un aumento en la temperatura corporal, hiperemia de las mucosas, disnea, secreción nasal serosa y lesiones erosivas en mucosa nasal. Este virus también es responsable de casos de vulvovaginitis pustular infeciosa, balanopositis pustular infecciosa y abortos (Oirschot, 1995; OIE, 2010).

Por último, el VRSB pertenece a la familia Paramyxoviridae y es un agente restringido al sistema respiratorio, presentando los animales afectados unas manifestaciones clínicas inespecíficas como: letargo, fiebre, tos, taquipnea y pérdida de apetito, que dificultan su diagnóstico clínico. La infección es más severa en animales jóvenes (Gershwin, 2007).

La falta de datos brasileños sobre la circulación de estos virus en los terneros (Gaeta et al. 2018) impide la formación de programas de vacunación efectivos y la determinación de las pérdidas económicas. Se planteó este estudio con el objetivo de evaluar la presencia de anticuerpos frente a VDVB, HVBo-1 y VRSB en terneros, con y sin enfermedad respiratoria, criados en el estado de Sao Paulo (Brasil) y su relación con la clínica que manifestaban.

\section{Materiales y métodos}

El presente trabajo fue realizado de acuerdo con los principios éticos de la investigación animal adoptados por el Comité de Ética para el uso de animales de la Facultad de Medicina Veterinaria y Zootecnia de la Universidad de Sao Paulo (\#Protocolo 2929/2013). Se estudiaron 63 terneros criados en una propiedad mediante un sistema semi-intensivo localizada en el estado de Sao Paulo. Los animales no estaban vacunados frente a VRSB, VDVB y HVBo-1. Los terneros eran inmediatamente separados de las madres después del nacimiento y el calostro / leche eran administrados artificialmente.

Los animales fueron sometidos a un examen físico, realizado de acuerdo con lo preconizado por Dirksen et al., (1993). Se evaluaron los parámetros vitales: la hidratación y coloración de las mucosas, la presencia de exudado nasal y tos, y se realizó la percusión torácica y auscultación pulmonar. A continuación, los terneros fueron clasificadas en sanos y enfermos o con síntomas de enfermedad respiratoria, cuando presentaban al menos dos de los siguientes parámetros: temperatura alta o equivalente a 39,5; presencia de exudado nasal mucopurulento / purulento; tos; ruidos pulmonares alterados y frecuencia respiratoria por encima de 40 movimientos respiratorios por minuto (Benesi et al., 2013; Gaeta et al., 2017a). Las muestras de sangre venosa se obtuvieron a través de la punción de la vena coccígea o yugular, utilizando tubos de vacío. Las muestras fueron centrifugadas a $600 \mathrm{x}$ g durante 15 minutos y el suero obtenido fue guardado en viales conservados a $-20^{\circ} \mathrm{C}$ hasta su procesado. La identificación de anticuerpos se realizó por medio de la técnica de neutralización de acuerdo con OIE (2010) y OIE (2015) para HVBo-1 y VDVB respectivamente y de acuerdo con Affonso et al. (2011) para VRSB. Cepas de VDVB-Ia NADL, cepa BoHV1 Los Ángeles y ATCC VR-1485 se utilizaron como controles positivos. Los títulos de anticuerpos se expresaron como el inverso de la dilución sérica que presentó efecto citopático. Muestras con títulos iguales o mayores a tres, se consideraron positivas. Los resultados se mostraron a través de frecuencias absolutas y relativas. El estudio de la asociación entre los hallazgos del examen físico y la presencia de anticuerpos fue realizado por medio de la prueba de la Chi-cuadrado o test exacto de Fisher. Las variables con $\mathrm{P}<0,05$ se consideraron significativas. Los valores entre $0,10<\mathrm{P}<0,05$ se consideraron tendencia estadística. Los análisis se realizaron con SPSS (SAS Institute Inc., NC).

\section{Resultados}

Se estudiaron 63 terneros pertenecientes a una granja que utiliza un sistema de producción semi-intensivo. Después del examen físico, los becerros fueron clasificados en sanos $(\mathrm{N}=43) \mathrm{y}$ con bronconeumonía $(\mathrm{N}=20)$. Los anticuerpos contra VDVB, VRSB y HVBo-1 fueron investigados por medio de la técnica 
de neutralización vírica. La frecuencia de anticuerpos contra VDVB, VRSB y HVBo-1 fue de 57,1\% (36/63), 93,7\% (59/63) y $66,7 \%(42 / 63)$ respectivamente. Entre los animales enfermos, se observó una mayor frecuencia de anticuerpos contra VRSB y HVBo-1 (Cuadro 1).

Cuadro 1: Porcentaje de terneros sanos y con bronconeumonía con serología positiva frente a VDVB, HVBo-1 y VRSB.

\begin{tabular}{cccc}
\hline Anticuerpos & $\begin{array}{c}\text { Sanos } \\
(\% ; \mathbf{N} / \mathbf{T})\end{array}$ & $\begin{array}{c}\text { Enfermos } \\
(\% ; \mathbf{N} / \mathbf{T})\end{array}$ & P-valor $(\mathbf{I C} \mathbf{9 5} \%)$ \\
\hline VDVB & & & \\
Ausente & $32,6(14 / 43)$ & $65,0(13 / 20)$ & $\mathbf{0 , 0 1}(\mathbf{0 , 0 8 5 - 0 , 7 9 6 )}$ \\
Presente & $67,4(29 / 43)$ & $35,0(07 / 20)$ & \\
VRSB & & & \\
Ausente & $02,3(01 / 43)$ & $15,0(03 / 20)$ & $0,09(0.013-1,390)^{*}$ \\
Presente & $97,7(42 / 43)$ & $85,0(17 / 20)$ & \\
& & & \\
HVBo-1 & & & \\
Ausente & $27,9(12 / 43)$ & $45,0(09 / 20)$ & $0,18(0,157-1,428)$ \\
Presente & $72.1(31 / 43)$ & $55.0(11 / 20)$ & \\
& & &
\end{tabular}

Además, se observó una asociación entre la presencia de anticuerpos contra VDVB y la presencia de bronconeumonía (P $=0,01$ ). En cuanto a las manifestaciones clínicas (Cuadro 2), se observó mayor frecuencia de anticuerpos contra VDVB en animales con exudado nasal seroso / mucoso $(\mathrm{P}=0,01)$.

Los animales deshidratados $(\mathrm{P}=0,008)$ y aquellos con una temperatura superior a $39,5^{\circ} \mathrm{C}(\mathrm{P}=0,03)$ presentaron mayor frecuencia de anticuerpos contra VRSB (Cuadro 3).

Las manifestaciones clínicas como tos y ruidos pulmonares alterados fueron numéricamente más prevalentes en animales con anticuerpos contra VRSB (Cuadro 3) o HVBo-1 (Cuadro 4). Asimismo, la frecuencia respiratoria por encima de 40 movimientos respiratorios por minuto fue más frecuente en animales con anticuerpos contra VDVB, HVBo-1 y VRSB.

Cuadro 2: Relación entre la presencia de anticuerpos anti-VDVB y las manifestaciones clínicas detectadas en los terneros.

\begin{tabular}{|c|c|c|c|}
\hline \multirow[b]{2}{*}{$\begin{array}{l}\text { Manifestaciones } \\
\text { clínicas }\end{array}$} & \multicolumn{2}{|c|}{ Anticuerpos } & \multirow[b]{2}{*}{ P-valor (IC 95\%) } \\
\hline & $\begin{array}{l}\text { VDVB } \\
\text { Ausente } \\
(\% ; \mathrm{N} / \mathrm{T})\end{array}$ & $\begin{array}{l}\text { VDVB } \\
\text { Presente } \\
(\% ; N / T)\end{array}$ & \\
\hline \multicolumn{4}{|l|}{ Actitud } \\
\hline Normal & $39,7(25 / 63)$ & $57,1(36 / 63)$ & \multirow{2}{*}{$0,18(-)$} \\
\hline Postrado & $03,2(02 / 63)$ & - & \\
\hline \multicolumn{4}{|l|}{ Deshidratación } \\
\hline Ausente & $85,2(23 / 27)$ & $85,7(31 / 36)$ & \multirow{2}{*}{$0,91(0,22-3,84)$} \\
\hline Presente & $14,8(04 / 27)$ & $13,9(36 / 36)$ & \\
\hline \multicolumn{4}{|l|}{ FR } \\
\hline$<40 \mathrm{rpm}$ & $70,4(19 / 27)$ & $50,0(16 / 59)$ & \multirow{2}{*}{$0,11(0,80-6,98)$} \\
\hline$>40 \mathrm{rpm}$ & $29,6(08 / 27)$ & $50,0(16 / 59)$ & \\
\hline \multicolumn{4}{|l|}{ Temperatura } \\
\hline$<39,5^{\circ} \mathrm{C}$ & $83,3(20 / 24)$ & $97,0(32 / 33)$ & \multirow[b]{2}{*}{$0,15(0,01-1,49)$} \\
\hline$>39,5^{\circ} \mathrm{C}$ & $16,7(04 / 24)$ & $03,0(01 / 33)$ & \\
\hline \multicolumn{4}{|l|}{ Tos } \\
\hline Ausente & $76,9(20 / 26)$ & $94,1(32 / 34)$ & \multirow{2}{*}{$0,06(0,03-1,13)^{*}$} \\
\hline Presente $^{\mathrm{a}}$ & $23,1(06 / 26)$ & $05,9(02 / 34)$ & \\
\hline \multicolumn{4}{|l|}{ Exudado nasal } \\
\hline Normal $^{b}$ & $38,1(08 / 21)$ & $73,9(17 / 23)$ & \multirow{2}{*}{$0,01(0,60-0,78)$} \\
\hline Alteradoc & $61,9(13 / 21)$ & $26,1(06 / 23)$ & \\
\hline \multicolumn{4}{|l|}{ Percusión } \\
\hline Normal & $68,8(11 / 16)$ & $94,1(16 / 17)$ & \multirow{2}{*}{$0,08(0,14-1,34) *$} \\
\hline Alterada ${ }^{\mathrm{d}}$ & $31,2(05 / 16)$ & $05,9(01 / 17)$ & \\
\hline \multicolumn{4}{|l|}{ Auscultación } \\
\hline Normal & $21,3(06 / 26)$ & $43,8(14 / 58)$ & \multirow{2}{*}{$0,09(0,12-1,21)^{*}$} \\
\hline Alterada ${ }^{e}$ & $76,9(20 / 26)$ & $56,2(18 / 58)$ & \\
\hline
\end{tabular}

Cuadro 3: Relación entre la presencia de anticuerpos anti-VRSB y las manifestaciones clínicas detectadas en terneros.

\begin{tabular}{|c|c|c|c|}
\hline \multirow[b]{2}{*}{$\begin{array}{l}\text { Manifestaciones } \\
\text { clínicas }\end{array}$} & \multicolumn{2}{|c|}{ Anticuerpos } & \multirow[b]{2}{*}{ P-valor (IC 95\%) } \\
\hline & $\begin{array}{l}\text { VRSB } \\
\text { Ausente (\%; } \\
\text { N/T) }\end{array}$ & $\begin{array}{c}\text { VRSB } \\
\text { Presente }(\% ; N / T)\end{array}$ & \\
\hline $\begin{array}{l}\text { Actitud } \\
\text { Normal }\end{array}$ & \multicolumn{3}{|c|}{ Actitud } \\
\hline Postrado & - & $03,4(02 / 59)$ & $1,00(-)$ \\
\hline \multicolumn{4}{|l|}{ Deshidratación } \\
\hline Ausente & $25,0(01 / 04)$ & $89,8(53 / 63)$ & \\
\hline Presente & $75,0(03 / 04)$ & $10,2(06 / 63)$ & $0,008(0,003-0,42)$ \\
\hline \multicolumn{4}{|l|}{ FR } \\
\hline$<40 \mathrm{rpm}$ & $50,0(02 / 04)$ & $60,0(33 / 55)$ & \multirow{2}{*}{$1,00(0,08-5,09)$} \\
\hline$>40 \mathrm{rpm}$ & $50,0(02 / 04)$ & $40,0(22 / 55)$ & \\
\hline \multicolumn{4}{|l|}{ Temperatura } \\
\hline$<39,5^{\circ} \mathrm{C}$ & $50,0(02 / 04)$ & $94,3(50 / 53)$ & \multirow[t]{2}{*}{$0,03 \quad 0,006-0,58)$} \\
\hline$>39,5^{\circ} \mathrm{C}$ & $50,0(02 / 04)$ & $05,7(03 / 53)$ & \\
\hline \multicolumn{4}{|l|}{ Tos } \\
\hline Ausente & $100(04 / 04)$ & $85,7(48 / 56)$ & \multirow{2}{*}{$1,00-)$} \\
\hline Presente $^{\mathrm{a}}$ & - & $14,3(08 / 56)$ & \\
\hline \multicolumn{4}{|l|}{ Exudado nasal } \\
\hline Normal $^{b}$ & $33,3(01 / 03)$ & $58,5(24 / 41)$ & \multirow{2}{*}{$0,57(0,03-4,22)$} \\
\hline Alteradoc & $66,7(02 / 03)$ & $41,5(17 / 41)$ & \\
\hline \multicolumn{4}{|l|}{ Percusión } \\
\hline Normal & $81,8(27 / 33)$ & $81,8(27 / 33)$ & \multirow{2}{*}{$(-)$} \\
\hline Alterada $^{\mathrm{d}}$ & $18,2(06 / 33)$ & $18,2(06 / 33)$ & \\
\hline \multicolumn{4}{|l|}{ Auscultación } \\
\hline Normal & $25,0(01 / 04)$ & $35,2(19 / 54)$ & \multirow{2}{*}{$1,00(0,06-6,31)$} \\
\hline Alterada ${ }^{e}$ & $75,0(03 / 4)$ & $64,8(35 / 54)$ & \\
\hline
\end{tabular}

* Tendencia estadistica: $0,05<\mathrm{P}<0,10$

Productiva no productiva, "geroso'mucoso; 'mucopurulento/ purulento; 'Submate /mate;

Roncuidos/sibilamcias/crepitaciones.

Cuadro 4: Relación entre la presencia de anticuerpos anti-HVBo-1 y las manifestaciones clínicas detectadas en los terneros.

\begin{tabular}{|c|c|c|c|}
\hline \multirow[b]{2}{*}{$\begin{array}{l}\text { Manifestaciones } \\
\text { clínicas }\end{array}$} & \multicolumn{2}{|c|}{ Anticuerpos } & \multirow[b]{2}{*}{ P-valor (IC 5\%) } \\
\hline & $\begin{array}{l}\text { HVBo-1 } \\
\text { Ausente } \\
(\% ; \mathrm{N} / \mathrm{T})\end{array}$ & $\begin{array}{l}\text { HVBo-1 } \\
\text { Presente } \\
(\% ; \mathrm{N} / \mathrm{T})\end{array}$ & \\
\hline \multicolumn{4}{|l|}{ Actitud } \\
\hline Normal & $95,2(20 / 21)$ & $97,6(41 / 42)$ & \multirow{2}{*}{$1,00(0,29-8,22)$} \\
\hline Postrado & $04,8(01 / 21)$ & $02,4(01 / 42)$ & \\
\hline \multicolumn{4}{|l|}{ Deshidratación } \\
\hline Ausente & $85,7(18 / 21)$ & $85,7(36 / 42)$ & \multirow[b]{2}{*}{$1,00(0,22-4,46)$} \\
\hline Presente & $14,3(03 / 21)$ & $14,3(06 / 42)$ & \\
\hline \multicolumn{4}{|l|}{ FR } \\
\hline$<40 \mathrm{rpm}$ & $70,0(14 / 20)$ & $53,8(21 / 39)$ & \multirow[b]{2}{*}{$0,23(0,63-6,28)$} \\
\hline$>40 \mathrm{rpm}$ & $30,0(06 / 20)$ & $46,2(18 / 39)$ & \\
\hline \multicolumn{4}{|l|}{ Temperatura } \\
\hline$<39,5^{\circ} \mathrm{C}$ & $88,9(16 / 18)$ & $92,3(36 / 39)$ & \multirow[b]{2}{*}{$0,64(0,10-4,38)$} \\
\hline$>39,5^{\circ} \mathrm{C}$ & $11,1(02 / 18)$ & $07,7(03 / 39)$ & \\
\hline \multicolumn{4}{|l|}{ Tos } \\
\hline Ausente & $94,7(18 / 19)$ & $82,9(34 / 41)$ & \multirow[b]{2}{*}{$0,41(0,42-32,5)$} \\
\hline Presente $^{\mathrm{a}}$ & $05,3(01 / 19)$ & $17,1(07 / 41)$ & \\
\hline \multicolumn{4}{|l|}{ Exudado nasal } \\
\hline Normal $^{b}$ & $40,0(06 / 15)$ & $35,5(19 / 29)$ & \multirow[b]{2}{*}{$0,10(0,09-1,26)$} \\
\hline Alteradoc & $60,0(09 / 15)$ & $34,5(10 / 29)$ & \\
\hline \multicolumn{4}{|l|}{ Percusión } \\
\hline Normal & $66,7(06 / 09)$ & $87,5(21 / 24)$ & \multirow[b]{2}{*}{$0,30(0,04-1,79)$} \\
\hline Alterada ${ }^{\mathrm{d}}$ & $33,3(03 / 09)$ & $12,5(03 / 24)$ & \\
\hline \multicolumn{4}{|l|}{ Auscultación } \\
\hline Normal & $31,6(06 / 19)$ & $64,1(25 / 39)$ & \multirow{2}{*}{$0,74(0,25-2,65)$} \\
\hline Alterada ${ }^{\mathrm{e}}$ & $68,4(13 / 19)$ & $35,9(03 / 39)$ & \\
\hline \multicolumn{4}{|c|}{$\begin{array}{l}\text { "Tendencia estadistica: } 0,05<\mathrm{P}<0,10 \text {. } \\
\text { "Productiváno productiva; } \\
\text { Ronquidos/sibilancias/crepitaciones. }\end{array}$} \\
\hline
\end{tabular}

Discusión

VDVB, VRSB y HVBo-1 están reconocidos como los principales agentes primarios de la enfermedad respiratoria bovina, con participación fundamental en el desarrollo de 
infecciones bacterianas secundarias por Pasteurella multocida, Mannheimia haemolytica, Histophilus somni y Mycoplasma bovis. El presente trabajo confirma la presencia de la circulación de estos virus en el rebaño analizado, pero la asociación entre estos agentes virales y la enfermedad respiratoria no ha sido detectada. Esta ausencia de asociación también fue observada por Gaeta et al. (2017b) estudiando becerros pertenecientes a explotaciones familiares, con producción del tipo extensivo.

La frecuencia de anticuerpos contra VDVB fue menor que la detectada en otros estados como Maranhão (Chaves et al., 2010), Rio Grande do Sul (Quincozes et al., 2007, Piovesan et al., 2013) y Goiás (Brito et al., 2010). Sin embargo, se encontraron mayores frecuencias de anticuerpos que los encontrados en un sistema extensivo (Gaeta et al., 2018). La presencia de anticuerpos frente al VRSB fue mayor que la detectada en otros estudios hechos en Brasil. En la región Sur, Arns et al. (2003) detectaron prevalencia del 75\%. En el Paraná, Sponchiado (2014) detectó el 22,3\% de animales positivos. En las comunidades rurales del Pontal del Paranapanema (São Paulo, Brasil), Gaeta et al. (2018) detectaron un 37,4\% de terneros con serología positiva. Por último, la frecuencia de anticuerpos contra HVBo-1 fue similar a las encontradas en los estados brasileños de Espírito Santo (Santos et al., 2014) y Maranhão (Chaves et al., 2012) y mayores que la encontrada en los asentamientos en el estado de São Paulo (Gaeta et al., 2018) y en Rio Grande do Sul (Becker et al., 2015). El muestreo, la edad de los animales y las distintas pruebas diagnósticas empleadas pueden estar relacionadas con los diferentes resultados encontrados en otros estudios (Coutinho et al., 2009).

Los sistemas de cría de tipo semi-intensivo o intensivo permiten la aglomeración de los animales, facilitando la transmisión de agentes infecciosos. Además, son explotaciones en las que se vacunan a las hembras antes del parto, garantizando la transferencia de inmunidad pasiva a los terneros, elevando el número de animales positivos, en comparación con los sistemas de producción extensivos. Sin embargo, el rebaño analizado no recibía vacuna contra los virus respiratorios. En un intento por obtener un diagnóstico presuntivo de los microorganismos relacionados con la enfermedad respiratoria bovina a partir de las manifestaciones clínicas observadas, se estudió la asociación de éstas con los anticuerpos frente a VRSB, VDVB y HVBo1 en los terneros. Se observó una tendencia estadística de asociación entre la presencia de anticuerpos contra VDVB y sonidos pulmonares anormales a la auscultación. La presencia de anticuerpos contra VDVB se asoció a la presencia de exudado nasal mucopurulenta / purulenta. En un trabajo similar, Gaeta et al. (2018) observaron la asociación de la misma manifestación clínica con anticuerpos contra el VRSB. Aunque son virus diferentes, se sabe que la presencia de exudado nasal seroso es característico de infecciones respiratorias de origen viral (Gonçalves, 2014).

\section{Conclusiones}

La investigación de la presencia directa o indirecta de los principales agentes relacionados con la enfermedad respiratoria de los bovinos es importante para el monitoreo de los rebaños y para la toma de decisiones en cuanto a las medidas de prevención y control. El presente trabajo muestra tasas de serología positiva superiores a las encontradas en otros estudios realizados en explotaciones con un sistema extensivo, confirmando la importancia del manejo en la transmisión de los microorganismos.

\section{Agradecimientos}

Los autores agradecen la Fundación de Amparo a la Investigación del Estado de São Paulo por el apoyo financiero (PROCESO FAPESP 2012 / 24928-0).

\section{Referencias bibliográficas}

Affonso, I. B., Gatti, S. P., Alexandrino, B., Oliveira, M. C., Medeiros, A. S. R., Buzinaro, M., Samara, S. I. (2011). Detection of antibodies against bovine respiratory syncytial virus (BRSV) in dairy cattle with different prevalences of bovine herpesvirus type 1 (BoHV-1) in São Paulo State, Brazil. Semina: Ciên Agr, 32(1), 295-300.

Arns. C.W., Campalans, J., Costa, S. C. B., Domingues, H. G., D'arce, R. C. F., Almeida, R. S., Coswig, L. T. (2003). Characterization of bovine respiratory syncytial virus isolated in Brazil. Braz J Med Bio Res, 36(2), 213-218.

Becker, A. S., Rodrigues, M. G., Orlandin, J. R., Menezes, P. Q., Matos, C. S., Wilsmann, D. E., Viana, A. E., Rodrigues, P. R. C. (2015). Anticorpos neutralizantes contra o herpesvirus bovino tipo 1 e o vírus da diarreia viral bovina em bovinos vacinados e não vacinados da região sul do estado do Rio Grande do Sul. Sci Ani Health, 3(2), 209-220.

Benesi, F. J., Bertagnon, H. G., Wachholz, L., Leal, M. L. R., Fernandes, W. R., Benites, N. R., Melville, P. A. (2013). Microbiota bacteriana e citologia da região traqueobrônquica de bezerros no período neonatal. Pesq Vet Bras, 33(6), 700-704.

Bosch, A. A. T. M., Biesbroek, G., Trzcinski, K., Sanders, E. A. M., Bogaert, D. (2013). Viral and Bacterial Interactions in the Upper Respiratory Tract. PLoS Pathogens, 9(1), e1003057.

Brito, W. M. E. D., Alfaia, B. T., Caixeta, S. P.,Ribeiro, A. C. C., Miranda, T. D. M. T., Barbosa, A. C. V. C., Barthasson, D. L., Linhares, D. C., Faria, B. O. (2010). Prevalência da infecção pelo Vírus da Diarréia Viral Bovina (BVDV) no Estado de Goiás, Brasil. Rev Pat Trop, 39(1), 07-20.

Chaves, N. P., Bezerra, D. C., Sousa, V. E., Santos, H. P., Pereira, H. M. (2010). Frequência de anticorpos e fatores de risco para a infecção pelo vírus da diarreia viral bovina em fêmeas bovinas leiteiras não vacinadas na região Amazônica Maranhense, Brasil. Ciênc. Rural, 40(6), 1448-1451.

Chaves, N. P., Bezerra, D. C., Sousa, V. E., Santos, H. P., Pereira, H. M. (2012) Frequência e fatores associados à infecção pelo vírus da diarreia viral bovina em bovinos leiteiros não vacinados no Estado do Maranhão. Arq Inst Biol, 79(4), 495-502.

Coutinho, A. S., Oliveira Filho, J. P., Silva, D. P. G., Oliveira, A. P., Marcondes, J. S., Chiacchio, S. B., Paes, A. C., Siqueira, A. K., Amorim, R. M., Gonçalves, R. C. (2009). Mannheimiose pulmonar experimental em bezerros: swab nasal e nasofaringeano como auxílio diagnóstico. Pesq Vet Bras, 29(1), 83-88.

Dirksen, G., Gruner, H. D., Stober, M. (1993). Exame Clínico dos Bovinos. Guanabara Koogan, $418 \mathrm{p}$.

Gaeta, N. C., Ribeiro, B. L. M., Alemán, M. A. R., Marques, E. C., Hellmeister, A. N., Pituco, E. M., Gregory, L. (2018). Serological investigation of antibodies against respiratory viruses in calves from Brazilian family farming and their relation to clinical signs of bovine respiratory disease. Pesq Vet Bras, 38(4), 642-648. 
Gaeta, N. C., Lima, S. F., Teixeira, A. G., Ganda, E. K., Oikonomou, G., Gregory, L., Bicalho, R. C. (2017a). Deciphering upper respiratory tract microbiota complexity in healthy calves and calves that develop respiratory disease using shotgun metagenomics. J Dairy Sci, 100(2), 1445-1458.

Gaeta, N. C., Ribeiro, B. L. M., Alemán, M. A. R., Thomazelli, L. M., Durigon, E. L., Nogueira, A. H. C., Stefano, E., Okuda, L. H., Pituco, E. M., Gregory, L. (2017b). Evaluation of bovine parainfluenza type3 virus and influenza virus D participation in bovine respiratory disease of calves from Brazilian family farming. Med Vet (Brazil), 11(4), 227-232.

Gershwin L. J. (2007). Bovine respiratory syncytial virus infection: immunopathogenic mechanisms. Anim Health Res Ver, 8, 207-213.

Gonçalves, R. C. (2014). Semiologia do sistema respiratório. En: Semiologia Veterinária: a arte do diagnóstico (pp. 313-331). São Paulo: Roca.

Lanyon, S. R., Hill, F. I., Reichel, M. P., Browlie, J. (2014). Bovine viral diarrhoea: patogenesis and diagnosis. Vet. J, 199(2), 201-209.

Miles, D. G. (2009). Overview of the North American beef cattle industry and the incidence of bovine respiratory disease (BRD). Anim Health Res Rev, 10, 101-103.

OIE (2018). Bovine viral diarrhoea. En: Manual of Diagnostic Tests and Vaccines for Terrestrial Animals. OIE Terrestrial Manual 2018 (pp. 1075-1096). [S.1.]: Organizaçao Internacional de Epizootias. Recuperado de: https://www.oie.int/fileadmin/Home/eng/Health_ standards/tahm/3.04.07_BVD.pdf

OIE (2018). Infectious bovine Rhinotracheitis/Infectious Pustular Vulvovaginitis. En: Manual of Diagnostic Tests and Vaccines for Terrestrial Animals. OIE Terrestrial Manual 2018 (pp. 11391157). [S.1.]: Organizaçao Internacional de Epizootias. Recuperado de: https://www.oie.int/fileadmin/Home/eng/Health_standards/ tahm/3.04.11_IBR_IPV.pdf

Oirschot, J. T. (1995). Bovine herpesvirus 1 in semen of bulls and the risk of transmission: an overview. Vet $Q, 17(1), 29-33$.

Piovesan, M., Fernandes, M. H. V., Corrêa, R. A., Prado, M. H. J., Camargo, A. D., Rodrigues, P. (2013). Anticorpos contre herpesvirus bovino tipo-1, vírus da diarreia viral bovina e virus da leucose enzoótica bovina na região da campanha do estado do Rio Grande do Sul. Sci Ani Health, 1(1), 38-49.

Quincozes, C. G., Fischer, G., Hubner, S. D. O., Vargas, G. D. A., Vidor, T., Brod, C. S. (2007). Prevalência e fatores associados à infecção pelo vírus da diarréia viral bovina na região Sul do Rio Grande do Sul. Semina: Cienc Agrárias, 28(2), 269-276.

Santos, M. R., Ferreira, H. C. C., Santos, M. A., Saraiva, G. L., Tafuri, N. F., Santos, G. M., Tobias, F. L., Moreira, M. A. S., Almeida, M. R., Silva Junior, A. (2014). Antibodies against Bovine herpesvirus 1 in dairy herds in the state of Espirito Santo, Brasil. Rev. Ceres, 61(2), 280-283.

Sponchiado, D. (2014). Prevalência dos principais vírus respiratórios em bovios da raça holandesa, no estado do Paraná (Tesis doctoral). Universidade Estadual Paulista Julio de Mesquita Filho, São Paulo. Recuperado de: https://repositorio.unesp.br/handle/11449/123337

USDA (2013). Feedlot 2011. Part IV: Health and Health Management on U.S. Feedlots with a capacity of 1,000 or more head. En: NAHMS Feedlot Studies. Fort Collins: National Animal Health Monitoring System. Recuperado de: https://www.aphis.usda.gov/animal_health/ nahms/feedlot/downloads/feedlot2011/Feed11_dr_PartIV.pdf

Nota de Contribución:

N. C. Gaeta $20 \%$

B. L. M. Ribeiro $16 \%$

M. A. R. Alemán 16\%

B. A. F. D. Oliveira $16 \%$

C. P. Ribeiro $16 \%$

L. Gregory $16 \%$ 